\title{
Características de crescimento, composição química, física e estimativa de massa seca de madeira em clones e espécies de Eucalyptus jovens
}

\author{
Growing characteristics, chemical composition, physical and dry mass estimated of wood in young \\ Eucalyptus species and clones
}

\author{
Paulo Fernando Trugilho ${ }^{\mathrm{I}}$ Selma Lopes Goulart ${ }^{\mathrm{II}}$ Claudinéia Olímpia de Assis ${ }^{\mathrm{II}}$ \\ Fernanda Bueno Sarro Couto ${ }^{\text {III }}$ Isabel Cristina Nogueira Alves ${ }^{\text {II }}$ \\ Thiago de Paula Protásio ${ }^{\mathrm{II}}$ Alfredo Napoli ${ }^{\mathrm{IV}}$
}

RESUMO

O objetivo deste trabalho foi avaliar o efeito do material genético, clones e espécies, e da classe de diâmetro nas características de crescimento, composição química, densidade básica e a estimativa de massa seca de madeira. Foram avaliados quatro clones e cinco espécies, aos quatro anos de idade, de Eucalyptus procedentes da empresa RIMA Industrial S.A. Foram avaliadas cinco árvores por clone e espécie em três e quatro classes diamétricas, respectivamente. Avaliaram-se as características individuais de crescimento da árvore, a densidade básica, a estimativa de massa seca, o teor de lignina, extrativos totais, holocelulose e cinzas. O volume, com e sem casca, e a estimativa de massa seca apresentaram efeito significativo de material genético (clone e espécie) e classe diamétrica. O teor de lignina não foi influenciado significativamente pelo material genético e classe diamétrica. A densidade básica apresentou efeito significativo de classe diamétrica para os clones e de material genético para as espécies. O teste de identidade de modelos somente foi não significativo para a estimativa de massa seca para as espécies, possibilitando usar a equação comum independente da espécie. Os clones apresentaram madeira mais homogênea, com menor densidade básica, extrativos totais e lignina total e maior teor de holocelulose, características mais adequadas à produção de polpa celulósica. As espécies apresentaram madeira mais heterogênea, com maior densidade básica, estimativa de massa seca, extrativos totais e lignina total e menor teor de cinzas e holocelulose, características desejáveis à produção de bioenergia.

Palavras-chave: qualidade, madeira jovem, eucalipto.

\section{ABSTRACT}

This research aimed to determine the basic density, the estimate of dry mass and the chemical composition of clones and Eucalyptus species. Four clones and five species of Eucalyptus were appraised, with four years old, coming from the RIMA Industrial Company S.A. Five trees were sampled for clone and species in three and four diametric classes, respectively. Wood characteristics as the basic density, the insoluble and soluble lignin in acid sulfuric, extractive total and ash content in the wood were available. The results showed that the volume, with or without bark, and estimated dry mass showed significant effects of genetic material (clone and species) and diameter class. The lignin content was not significantly influenced by both genetic material and diameter class. Wood basic density had a significant effect of diameter class for clones and genetic material for the species. The identity test for linear models was only not significant for estimated dry mass of the species, indicating to be possible to use the common equation independent of the species. The clones showed a more homogeneous wood with lower average basic density, extractives and total lignin content and higher holocellulose content, characteristics which are suitable for pulp production. The wood species showed more heterogeneous with highest average basic density, estimated dry weight, total lignin and extractives content and lower ash and holocellulose content, characteristics desirable for bioenergy production.

Key words: quality, young wood, eucalypts.

\section{INTRODUÇÃO}

A madeira é um material heterogêneo, tanto do ponto de vista físico, químico, mecânico e anatômico (BROWNING, 1963). Essa heterogeneidade está relacionada com as variações

'Departamento de Ciências Florestais, Universidade Federal de Lavras (UFLA), 37200-00, Lavras, MG, Brasil. E-mail: trugilho@dcf.ufla.br. Autor para correspondência.

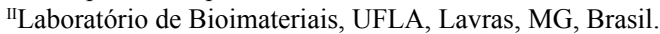

IIIDepartamento de Pesquisa Florestal, Rima Industrial S.A., Belo Horizonte, MG, Brasil.

${ }^{\mathrm{IV}}$ Centre de Coopération Internationale en Recherche Agronomique pour le Développement (CIRAD), Montpellier, França. Recebido 07.05.13 Aprovado 03.09.14 Devolvido pelo autor 11.11.14 CR-2013-0625.R1 
nos sentidos radial e longitudinal que a madeira normalmente apresenta, em função especialmente da diferenciação dos tipos de células e tecidos. Apesar de dificultar a utilização, essa variabilidade permite que se encontrem diferentes materiais para atender a distintas utilizações, fazendo da madeira um notável material. ZOBEL E JETT (1995) relataram que a alta variabilidade do gênero Eucalyptus, em termos de número de materiais genéticos existentes e associados a amplas diferenças tecnológicas da madeira, permite que inúmeras aplicações sejam possíveis com esta matéria-prima.

Variações na madeira ocorrem devido à idade, ao material genético, sítio, espaçamento, nutrição e interações ambientais. Geralmente, as características da madeira tendem a estabilizar com o aumento da idade da árvore. Dentro do anel de crescimento, as características tendem a aumentar do lenho inicial para o tardio (DOWNES et al., 1997). Este mesmo efeito ocorre do lenho juvenil para o maduro ou adulto. Diferenças entre materiais genéticos são marcantes, bem definidas e peculiares dentro do gênero Eucalyptus. A qualidade do sítio afeta diretamente a produtividade da floresta e, segundo LARSON et al. (2001), pode provocar variação na formação e crescimento do lenho, modificando a qualidade da madeira.

A qualidade da madeira é o resultado das características físicas e químicas apresentadas por uma árvore ou uma de suas partes que lhe permite satisfazer os requisitos de propriedades para diferentes produtos finais (MITCHELL, 1961). Os estudos de qualidade objetivam inferir sobre as relações entre características e o produto final desejado. Assim, é necessário que diferentes características sejam determinadas, que suas variações sejam conhecidas e que a sua relação com o produto desejado seja devidamente estabelecida.

A determinação das propriedades físicas e químicas é importante do ponto de vista da utilização da madeira como fonte de energia, para produção de celulose e papel, painéis ou para a sua conversão em um produto combustível, utilizando-se, por exemplo, a carbonização ou pirólise. Avaliar os efeitos que podem influenciar a qualidade da madeira é necessário para conhecer as variações sofridas pelas diferentes propriedades, tanto nas espécies como nos clones plantados industrialmente.

SANTANA et al. (2012), avaliando o efeito da idade e da classe diamétrica nas características da madeira de um clone de eucalipto plantado no sul de Minas Gerais, verificaram que a idade influenciou significativamente nas propriedades físicas, químicas e térmicas da madeira, exceto o teor de holocelulose, carbono e oxigênio elementares. Observaram, também, que a classe diamétrica exerceu grande influência sobre as características da madeira.

O objetivo do presente trabalho foi avaliar o efeito do material genético, clones e espécies, e da classe de diâmetro nas características de crescimento, composição química, densidade básica e a estimativa de massa seca de madeira de clones e espécies de Eucalyptus.

\section{MATERIAL E MÉTODOS}

Foram avaliados quatro clones e cinco espécies de Eucalyptus procedentes da empresa RIMA Industrial S.A. Foram avaliadas cinco árvores por material genético, todas com quatro anos de idade. Todas as árvores foram devidamente cubadas para determinação do seu volume individual com e sem casca do fuste comercial, de acordo com a equação de Smalian. As classes diamétricas foram definidas considerando a medição de 100 árvores nos talhões amostrados. Para os clones, foram definidas as classes de 11,13 e $15 \mathrm{~cm}$ e, para as espécies, além dessas, a de $17 \mathrm{~cm}$.

As árvores selecionadas foram derrubadas e a amostragem consistiu da retirada de discos, de aproximadamente $2,5 \mathrm{~cm}$ de espessura, em cinco pontos longitudinais ao tronco, ou seja, na base $(0 \%), 25 \%, 50 \%, 75 \%$ e $100 \%$ da altura comercial da árvore.

Os discos foram devidamente numerados e encaminhados ao Laboratório de Tecnologia da Madeira do Departamento de Ciências Florestais da Universidade Federal de Lavras para a determinação das características da madeira. No Laboratório, estes foram, então, seccionados em quatro cunhas sempre passando pela medula. Duas cunhas opostas foram utilizadas na determinação da densidade básica da madeira, conforme a norma NBR 11941 da ASSOCIAÇÃO BRASILEIRA DE NORMAS TÉCNICAS (2003). A densidade básica média da árvore foi considerada como sendo a média aritmética (DBm) dos pontos longitudinais de amostragem em relação ao tronco das árvores.

Com o volume individual sem casca (Vsc $\mathrm{em}^{3}$ ) e a densidade básica média (DBm em $\mathrm{kg} \mathrm{m}^{-3}$ ), foi estimada a massa seca individual das árvores (MS em $\mathrm{kg})$.

Para a determinação da composição química da madeira, foram utilizadas amostras retiradas das outras duas cunhas opostas. Foram retirados materiais de todas as posições longitudinais 
de amostragem para a formação de uma amostra composta. O teor de lignina insolúvel (Klason) foi obtido de acordo com a metodologia proposta por GOMIDE \& DEMUNER (1986). O teor de lignina solúvel (Klason) em ácido sulfúrico foi determinado de acordo com a metodologia proposta por GOLDSCHIMID (1971). O teor de lignina total foi considerado como sendo a soma da lignina insolúvel com a solúvel.

Os teores de cinzas e extrativos da madeira foram determinados, respectivamente, de acordo com as normas m 11/77 e M 3/89 da ASSOCIAÇÃO BRASILEIRA TÉCNICA DE CELULOSE E PAPEL-ABTCP (1974). O teor de holocelulose foi determinado pela análise somatória.

$\mathrm{Na}$ avaliação do experimento, foi adotado o delineamento experimental inteiramente casualisado, disposto em esquema fatorial com dois fatores, clone ou espécie e classe diamétrica, com cinco repetições. Especificamente para o diâmetro a $1,30 \mathrm{~m}$ de altura do solo (DAP) e altura total das árvores, foi utilizado o delineamento inteiramente casualisado com cinco repetições. $\mathrm{Na}$ avaliação do efeito de material genético (clone ou espécie), foi aplicado o teste de Tukey, em nível de 5\% de probabilidade, enquanto que, na avaliação do efeito de classe diamétrica (efeito quantitativo), foi utilizada a análise de regressão.

Para as características volume, com e sem casca, densidade básica e estimativa de massa seca, foi estimada uma equação de regressão por material genético (clone e espécie), onde foi aplicado o teste de identidade de modelos lineares, conforme descrito por GRAYBILL (1976), para verificar se existe uma equação comum, modelo linear simples, para clones ou espécies em função da classe diamétrica.

\section{RESULTADOS E DISCUSSÃO}

A análise de variância para as características de crescimento da árvore dos clones e espécies mostrou que os efeitos de clone e espécie foram não significativos para o DAP, entretanto, a altura total e o volume, com e sem casca, apresentaram significância em nível de $1 \%$ de probabilidade. $\mathrm{O}$ volume, com e sem casca, também apresentou significância para o efeito de classe diamétrica, tanto nos clones como nas espécies. $\mathrm{O}$ efeito da interação clone/espécie x classe diamétrica foi não significativo. A característica porcentagem de casca apresentou efeito significativo somente para o efeito de espécie. De modo geral, os clones apresentaram menor DAP e porcentagem de casca e maior altura total, enquanto que o volume, com e sem casca, não apresentou grandes diferenças entre clones e espécies.

Verificou-se, ainda, que os coeficientes de variação experimental (CVe) foram baixos, especialmente para os clones, indicando que existe uma menor variabilidade nesse tipo de material genético, para as características de crescimento da árvore, e que o delineamento utilizado e a qualidade da coleta dos dados foram adequados.

Os valores médios e o teste e comparação múltipla realizado para as características de crescimento da árvore, para os efeitos principais de clone e espécie, encontram-se na (Tabela 1). De modo geral, os clones apresentaram árvores mais altas, com

Tabela 1 - Valores médios e teste de comparação múltipla realizado para as características de crescimento da árvore.

\begin{tabular}{|c|c|c|c|c|c|}
\hline CLONE & $\mathrm{DAP}(\mathrm{cm})$ & $\mathrm{Ht}(\mathrm{m})$ & $\operatorname{Vcc}\left(\mathrm{m}^{3}\right)$ & $\operatorname{Vsc}\left(\mathrm{m}^{3}\right)$ & CASCA $(\%)$ \\
\hline 1 & $12,44 \mathrm{a}$ & $20,62 \mathrm{ab}$ & $0,12242 \mathrm{a}$ & $0,10260 \mathrm{ab}$ & $16,29 \mathrm{a}$ \\
\hline 2 & $12,34 \mathrm{a}$ & $18,40 \mathrm{c}$ & $0,10534 \mathrm{~b}$ & $0,08812 \mathrm{c}$ & $15,99 \mathrm{a}$ \\
\hline 3 & $12,46 \mathrm{a}$ & $21,36 \mathrm{a}$ & $0,12264 \mathrm{a}$ & 0,10448 a & $14,76 \mathrm{a}$ \\
\hline 4 & $12,36 \mathrm{a}$ & $19,32 \mathrm{bc}$ & $0,11046 \mathrm{~b}$ & $0,09356 \mathrm{bc}$ & $15,39 \mathrm{a}$ \\
\hline Média & 12,40 & 19,93 & 0,1152 & 0,0972 & 15,61 \\
\hline CVe (\%) & 10,45 & 4,64 & 3,74 & 4,71 & 7,41 \\
\hline ESPÉCIE & $\mathrm{DAP}(\mathrm{cm})$ & $\mathrm{Ht}(\mathrm{m})$ & $\operatorname{Vcc}\left(\mathrm{m}^{3}\right)$ & $\operatorname{Vsc}\left(\mathrm{m}^{3}\right)$ & CASCA $(\%)$ \\
\hline $\mathrm{EC}$ & $13,20 \mathrm{a}$ & $15,42 \mathrm{c}$ & $0,09770 \mathrm{~b}$ & $0,07294 \mathrm{~b}$ & $25,53 \mathrm{a}$ \\
\hline $\mathrm{EU}_{1}$ & $13,28 \mathrm{a}$ & $18,44 \mathrm{ab}$ & $0,11684 \mathrm{ab}$ & $0,09492 \mathrm{a}$ & $18,77 \mathrm{~b}$ \\
\hline $\mathrm{EU}_{2}$ & $13,24 \mathrm{a}$ & $17,62 \mathrm{~b}$ & $0,11024 \mathrm{ab}$ & $0,08986 \mathrm{ab}$ & $18,56 \mathrm{~b}$ \\
\hline $\mathrm{HB}_{1}$ & $13,16 \mathrm{a}$ & $18,62 \mathrm{ab}$ & $0,12218 \mathrm{a}$ & $0,10016 \mathrm{a}$ & $18,33 \mathrm{~b}$ \\
\hline $\mathrm{HB}_{2}$ & $13,10 \mathrm{a}$ & $19,90 \mathrm{a}$ & $0,13020 \mathrm{a}$ & $0,10568 \mathrm{a}$ & $19,58 \mathrm{~b}$ \\
\hline Média & 13,20 & 18,00 & 0,1154 & 0,0927 & 20,15 \\
\hline CVe (\%) & 16,49 & 5,42 & 6,96 & 7,93 & 10,71 \\
\hline
\end{tabular}

Valores médios, para clones e espécies, seguidos de mesma letra, na coluna, não diferem entre si pelo teste de Tukey, em nível de $5 \%$ de probabilidade. $\mathrm{CVe}=$ coeficiente de variação experimental. 
menores diâmetros (DAP) e menor porcentagem de casca. Entre as espécies, o Eucalyptus cloeziana (EC) apresentou a menor altura e volume, com e sem casca, e a maior porcentagem de casca no fuste.

Aanálise de variância para as características físicas e químicas da madeira dos clones e espécies mostrou que o efeito de clone foi significativo apenas para as características estimativa de massa seca, extrativos totais e cinzas. Para o efeito de espécie, apenas os teores de extrativos totais e lignina na madeira foram não significativos. De modo geral, a madeira dos clones apresentou menor valor médio de densidade básica, estimativa de massa seca, teor de extrativos totais e de lignina na madeira, enquanto que as espécies apresentaram os menores teores de cinzas e holocelulose.

Observou-se, também, que os coeficientes de variação experimental (CVe) foram de baixa magnitude, exceto para o teor de extrativos totais e cinzas na madeira. TRUGILHO et al. (2007 e 2012), trabalhando com diversos clones de Eucalyptus, aos 5,8 anos de idade, encontraram variação elevada para os teores de cinzas e extrativos na madeira, sendo o teor de extrativos na madeira o que apresentou a maior variabilidade entre os clones. Também podese verificar que os clones apresentaram os menores valores de $\mathrm{CVe}$, exceto para o teor de lignina e holocelulose, indicando possuírem madeira com maior homogeneidade, mesmo fato ocorrido nas características de crescimento da árvore.

SANTANA et al. (2012), estudando um clone de híbrido do Eucalyptus grandis e $\boldsymbol{E}$. urophylla, em diferentes idades e classes diamétricas, encontraram o mesmo resultado para o efeito da classe diamétrica sobre a composição química, densidade básica e estimativa de massa seca de madeira.

Os valores médios e o teste e comparação múltipla realizado para as características físicas e químicas da madeira para os efeitos principais de clone e espécie encontram-se na (Tabela 2). Os resultados das análises químicas e densidade básica da madeira dos clones e espécies estão em conformidade com os obtidos por TRUGILHO et al. (1996), TRUGILHO (2009) e NEVES et al. (2011).

Pela tabela 2, verifica-se que o clone 1 foi o que apresentou o maior valor de densidade básica, estimativa de massa seca e teor de holocelulose; o clone 4, o maior teor de extrativos totais; os clones 2 e 3 , os maiores teores de lignina total; e o clone 3 , o maior teor de cinzas na madeira. Os clones 2 e 3 , por apresentarem valor intermediário de densidade básica, elevado teor de lignina e baixo de cinzas, possuem potencial para produção de bioenergia.

Para as espécies, verifica-se que o Eucalyptus cloeziana (EC) foi quem apresentou o maior valor médio de densidade básica e teor de lignina total; o híbrido $2\left(\mathrm{HB}_{2}\right)$, a maior estimativa de massa seca; o híbrido $1\left(\mathrm{HB}_{1}\right)$, o menor teor de holocelulose na madeira. O Eucalyptus urophylla (1 e 2) foi a espécie de menores valores de densidade básica, mesmo teor de lignina, entretanto, com diferentes teores de extrativos totais e cinzas. O EC e o $\mathrm{HB}_{1}$, por apresentarem os maiores valores de densidade básica e lignina total e baixo teor de cinzas,

Tabela 2 - Valores médios das características da madeira dos materiais genéticos avaliados.

\begin{tabular}{|c|c|c|c|c|c|c|}
\hline Clone & $\mathrm{DB}\left(\mathrm{g} \mathrm{cm}^{-3}\right)$ & MS (kg) & TET (\%) & TLIG (\%) & $\mathrm{CNz}(\%)$ & HOLO (\%) \\
\hline 1 & $0,466 \mathrm{a}$ & $47,84 \mathrm{a}$ & $4,72 \mathrm{~b}$ & $29,09 \mathrm{a}$ & $0,28 \mathrm{a}$ & $65,90 \mathrm{a}$ \\
\hline 2 & $0,452 \mathrm{a}$ & $40,42 \mathrm{~b}$ & $5,16 \mathrm{~b}$ & $30,09 \mathrm{a}$ & $0,14 \mathrm{~b}$ & $64,62 \mathrm{a}$ \\
\hline 3 & $0,444 \mathrm{a}$ & $46,43 \mathrm{a}$ & $5,52 \mathrm{ab}$ & $30,36 \mathrm{a}$ & $0,30 \mathrm{a}$ & $63,82 \mathrm{a}$ \\
\hline 4 & $0,430 \mathrm{a}$ & $40,45 \mathrm{~b}$ & $7,43 \mathrm{a}$ & $27,97 \mathrm{a}$ & $0,29 \mathrm{a}$ & $64,30 \mathrm{a}$ \\
\hline Média & 0,448 & 43,78 & 5,71 & 29,38 & 0,25 & 64,66 \\
\hline $\mathrm{CVe}(\%)$ & 4,06 & 5,03 & 16,97 & 6,00 & 13,06 & 2,79 \\
\hline Espécie & $\mathrm{DB}\left(\mathrm{g} \mathrm{cm}^{-3}\right)$ & MS (kg) & TET (\%) & LIG (\%) & $\mathrm{CNz}(\%)$ & HOLO (\%) \\
\hline $\mathrm{EC}$ & $0,590 \mathrm{a}$ & $43,58 \mathrm{ab}$ & $7,77 \mathrm{a}$ & $32,28 \mathrm{a}$ & $0,18 \mathrm{~b}$ & $59,78 \mathrm{c}$ \\
\hline $\mathrm{EU}_{1}$ & $0,460 \mathrm{~b}$ & $43,64 \mathrm{ab}$ & $7,45 \mathrm{a}$ & $30,31 \mathrm{a}$ & $0,18 \mathrm{~b}$ & $62,07 \mathrm{bc}$ \\
\hline $\mathrm{EU}_{2}$ & $0,446 \mathrm{~b}$ & $40,62 \mathrm{~b}$ & $4,50 \mathrm{a}$ & $30,31 \mathrm{a}$ & $0,36 \mathrm{a}$ & $64,84 \mathrm{a}$ \\
\hline $\mathrm{HB}_{1}$ & $0,484 \mathrm{~b}$ & $47,93 \mathrm{ab}$ & $8,95 \mathrm{a}$ & $31,16 \mathrm{a}$ & $0,11 \mathrm{~b}$ & $59,77 \mathrm{c}$ \\
\hline $\mathrm{HB}_{2}$ & $0,497 \mathrm{ab}$ & $51,31 \mathrm{a}$ & $7,06 \mathrm{a}$ & $29,72 \mathrm{a}$ & $0,17 \mathrm{~b}$ & $63,04 \mathrm{ab}$ \\
\hline Média & 0,495 & 45,42 & 7,14 & 30,76 & 0,20 & 61,90 \\
\hline $\mathrm{CVe}(\%)$ & 7,57 & 7,16 & 23,21 & 4,12 & 13,26 & 1,64 \\
\hline
\end{tabular}

$\mathrm{DB}=$ densidade básica, TET $=$ teor de extrativos totais, TLIG = teor de lignina total, $\mathrm{CNz}=$ teor de cinzas. EC = Eucalyptus cloeziana, $\mathrm{HB}$ = híbrido do $\boldsymbol{E}$. urophylla $\times$ E grandis, EU = Eucalyptus urophylla . Valores médios, para clone e espécie, seguidos de mesma letra, na coluna, não diferem entre si pelo teste de Tukey, em nível de $5 \%$ de probabilidade. 
possuem potencial para produção de bioenergia. O $\mathrm{HB}_{2}$ pode também ser considerado como potencial para essa atividade, por apresentar uma maior estimativa de massa seca.

Considerando que os baixos teores de extrativos e lignina e elevados teores de holocelulose são desejáveis para a produção de polpa celulósica e que, segundo GOMIDE et al. (2010), elevados teores de extrativos e lignina na madeira afetam negativamente o rendimento em polpa celulósica, pode-se afirmar que os clones apresentaram madeira com melhor potencial para esta atividade. Entretanto, altos valores de densidade básica e teor de lignina na madeira, associado com baixos valores de cinzas e holocelulose, são mais desejáveis para a produção de bioenergia (TRUGILHO et al., 1997; TRUGILHO et al., 2001; TRUGILHO, 2009; PROTÁSIO et al., 2012). Assim, as espécies apresentaram maior potencial para a produção de bioenergia.

Para a produção de carvão vegetal, esperase que, quanto maior o teor de holocelulose, menor será o rendimento e a qualidade do carvão vegetal (PROTÁSIO et al., 2012). Este fato está relacionado à menor resistência à degradação térmica das hemiceluloses e celulose (SHAFIZADEH, 1985).

A (Tabela 3) apresenta as equações ajustadas para o efeito de classe diamétrica dos clones e espécies para o volume, com e sem casca, densidade básica e estimativa de massa seca. Verifica-se que o volume, com e sem casca, apresentou elevada relação funcional positiva com a classe diamétrica, o que era esperado. É de conhecimento geral que o diâmetro afeta consideravelmente a produção volumétrica de uma floresta.

Pela (Tabela 3), observa-se que existe uma relação funcional positiva entre a densidade básica da madeira e a estimativa de massa seca com a classe diamétrica, tanto para os clones como para as espécies. TRUGILHO et al. (2010), avaliando a madeira de um clone de híbrido do $\boldsymbol{E}$. urophylla com o
E. grandis com seis anos de idade, observaram efeito significativo da classe diamétrica para a densidade básica e estimativa de massa seca. Os autores também verificaram uma tendência de aumento dessas propriedades com o incremento da classe diamétrica, corroborando o observado neste trabalho.

O resultado da análise de identidade de modelos lineares mostrou que, para os clones, a hipótese de igualdade das equações de regressão foi rejeitada para todas as características consideradas, ou seja, indicando que se devem utilizar equações ajustadas por clone. Entretanto, para as espécies, a hipótese de igualdade dos modelos não foi rejeitada para a estimativa de massa seca, ou seja, pode-se utilizar a equação comum independente da espécie.

\section{CONCLUSÃO}

O volume, com e sem casca, e a estimativa de massa seca apresentaram efeito significativo de material genético (clone e espécie) e classe diamétrica. Já o teor de lignina na madeira não foi influenciado significativamente, tanto pelo material genético como classe diamétrica. A densidade básica da madeira apresentou relação funcional positiva com a classe diamétrica para os clones, enquanto que a estimativa de massa seca apresentou relação funcional positiva com a classe diamétrica para os clones e espécies.

$\mathrm{O}$ teste de identidade de modelos lineares somente foi não significativo para a estimativa de massa seca para as espécies, indicando que se pode usar a equação comum independente das espécies. Os clones apresentaram madeira mais homogênea ou de menor variabilidade, com menor valor médio de densidade básica, extrativos totais e lignina total e maior teor de holocelulose.

As espécies apresentaram madeira mais heterogênea ou de maior variabilidade, com maior valor médio de densidade básica, estimativa de massa seca, extrativos totais e lignina total e menor teor de

Tabela 3 - Equações ajustadas para o efeito de classe diamétrica dos clones e espécies para o volume com e sem casca.

\begin{tabular}{|c|c|c|c|c|}
\hline \multirow{2}{*}{ Prop } & \multirow[b]{2}{*}{ Clone } & \multirow[b]{2}{*}{$r^{2}$} & \multirow[b]{2}{*}{ Espécie } & \multirow[b]{2}{*}{$r^{2}$} \\
\hline & & & & \\
\hline Vec & $\mathrm{Vcc}=-0,125358+0,0186 \mathrm{CD}$ & $99,49 * *$ & $\mathrm{Vcc}=-0,11528+0,0167 \mathrm{CD}$ & $99,43 * *$ \\
\hline Vsc & $\mathrm{Vsc}=-0,102617+0,0154 \mathrm{CD}$ & $99,69 * *$ & $\mathrm{Vsc}=-0,09781+0,0138 \mathrm{CD}$ & $99,50 * *$ \\
\hline DB & $\mathrm{DB}=0,3185+0,00975 \mathrm{CD}$ & $92,19 * *$ & $\mathrm{DB}=0,485155+0,000555 \mathrm{CD}$ & $2,65 \mathrm{~ns}$ \\
\hline MS & $\mathrm{MS}=-56,4845+7,7356 \mathrm{CD}$ & $99,83 * *$ & $\mathrm{MS}=-46,3980+6,6400 \mathrm{CD}$ & $99,60 * *$ \\
\hline
\end{tabular}

Prop = propriedade, Vcc e Vsc = volume com e sem casca, CD = classe diamétrica, $* *$ significativo a $1 \%$ de probabilidade. $\mathrm{DB}=$ densidade básica, $\mathrm{MS}$ = estimativa de massa seca, ns e ** não significativo e significativo a $1 \%$ de probabilidade. 
cinzas e holocelulose. O Eucalyptus cloeziana, por apresentar maior teor de lignina e densidade básica, foi o destaque, considerando o uso energético da madeira.

\section{AGRADECIMENTOS}

Os autores agradecem ao Conselho Nacional de Desenvolvimento Científico e Tecnológico (CNPq), Fundação de Amparo à Pesquisa do estado de Minas Gerais (FAPEMIG) e à empresa RIMA, Industrial S.A., pelo auxílio na realização do trabalho.

\section{REFERÊNCIAS}

ASSOCIAÇÃO BRASILEIRA DE NORMAS TÉCNICAS (ABNT). Normas técnicas NBR 11941. Madeira- Determinação da densidade básica. Rio de Janeiro, 2003. 6p.

\section{ASSOCIAÇÃO BRASILEIRA TÉCNICA DE CELULOSE E} PAPEL (ABTCP). Normas técnicas. São Paulo, 1974. 6p.

BROWNING, B.L. The chemistry of Wood. New York: John Wiley \& Sons, 1963. 689p.

DOWNES, G.M. et al. Sampling plantation eucalypts for wood and fibre properties. Austrália: CSIRO, 1997. 1342p.

GOLSCHIMID, O. Ultraviolet spectra. In: SARKANEN, K.V.; LUDWIG, C.H. Lignins: occurrence, formation, structure and reations. New York: John Wiley \& Sons, 1971. p.241-266.

GOMIDE, J.L.; DEMUNER, B.J. Determinação do teor de lignina em material lenhoso: método Klason modificado. O Papel. São Paulo, v.47, n.8, p.36-38, 1986

GOMIDE, J.L. et al. Análise de critérios de qualidade da madeira de eucalipto para produção de celulose Kraft. Revista Árvore, Viçosa, v.34, n.2, p.339-344, 2010. Disponível em: <http://www. scielo.br/pdf/rarv/v34n2/v34n2a17.pdf $>$. Acesso em 01 de out. de 2014.

GRAYBILL, F.A. Theory and application of the linear model Massachussets: Duxburt, 1976. 704p.

LARSON, P.R. et al. Formation and properties of juvenile wood in southern pines: a synopsis. Madison: USDA, 2001. 42p.

MITCHELL, H.L. A concept of intrinsic wood quality and nondestructive methods for determining quality in standing timber. Madison, Wisconsin: Forest Products Laboratory, 1961. Report n.2233.

NEVES, T.A. et al. Avaliação de clones de Eucalyptus em diferentes locais visando à produção de carvão vegetal. Pesquisa Florestal Brasileira, Colombo, v.31, n.68, p.319-330, 2011. Disponível em: <http://www.cnpf. embrapa.br/pfb/index.php/pfb/article/view/275/231>. Acesso em: 4 de set. de 2013. doi.org/10.4336/2011.pfb.31.68.319.
PROTÁSIO, T.P. et al. Análise de correlação canônica entre características da madeira e do carvão vegetal de Eucalyptus. Scientia Forestalis, Piracicaba, v.40, n.95, p.317-326, 2012. Disponível em: <http://www.ipef.br/publicacoes/scientia/nr95/ cap02.pdf $>$. Acesso em: 16 de set. de 2013.

SANTANA, W.M.S. et al. Effect of age and diameter classon the properties of wood from clonal Eucalyptus. Cerne, Lavras, v.18, n.1, p.1-8, 2012. Disponível em: <http://www.scielo.br/ scielo.php?pid=S0104-77602012000100001\&script=sci_arttext $>$. Acesso em: 15 de nov. de 2013. doi.org/10.1590/S010477602012000100001

SHAFIZADEH, F. Pyrolytic reactions and products of biomass. In: OVEREND, R.P. Fundamentals of biomass thermochemical conversion. London: Elsevier, 1985. p.183-217.

TRUGILHO, P.F. Densidade básica e estimativa de massa seca e lignina na madeira em espécies de Eucalyptus. Ciência e Agrotecnologia, Lavras, v.33, n.5, p.1228-1239, 2009. Disponível em: <http://www.scielo.br/pdf/cagro/v33n5/v33n5a05. pdf $>$. Acesso em: 16 de set. de 2013. doi.org/10.1590/S141370542009000500005 .

TRUGILHO, P.F. et al. Influência da idade nas características físico-químicas da madeira de Eucalyptus saligna. Cerne, Lavras, v.2, n.1, p.94-111, 1996. Diponível em: <http://www.dcf.ufla.br/ cerne/artigos/16-02-20099844v2_n1_artigo\%2009.pdf>. Acesso em: 15 de set. 2013

TRUGILHO, P.F. et al. Avaliação de clones de Eucalyptus para a produção de carvão vegetal. Cerne, Lavras, v.7, n.2, p.104-114, 2001. Disponível em: $<$ http://www.dcf.ufla.br/cerne/artigos/16-0220099844v2_n1_artigo\%2009.pdf>. Acesso em: 15 de set. de 2013.

TRUGILHO, P.F. et al. Aplicação da análise de correlação canônica na identificação de índices de qualidade da madeira de eucalipto para a produção de carvão vegetal. Revista Árvore, Viçosa, v.21, n.2, p.259-267, 1997.

TRUGILHO, P.F. et al. Qualidade da madeira de clones de espécies e híbridos naturais de Eucalyptus. Scientia Forestalis, Piracicaba, n.73, p.55-62, 2007. Disponível em: <http://www.ipef. br/publicacoes/scientia/nr73/cap06.pdf>. Acesso em: 2 de set. de 2013.

TRUGILHO, P.F. et al. Caracterização tecnológica da madeira de diferentes materiais genéticos de eucalipto. In: ENCONTRO BRASILEIRO EM MADEIRAS E EM ESTRUTURAS DE MADEIRA (EBRAMEM), 13p. 2012, Vitória-ES. Anais... Vitória: EBRAMEM, 2012. 1 CD-ROM

TRUGILHO, P.F. et al. Estimativa de carbono fixado na madeira de um clone híbrido de Eucalyptus urophylla e Eucalyptus grandis. Cerne, Lavras, v.16, supl., p.33-40, 2010. Disponível em: <http://www.def.ufla.br/ebramem.htm $>$. Acesso em: 04 de jan. 2013 .

ZOBEL, B.J.; JETT, J.B. Genetic of wood production. Berlin, Springer-Verlag, 1995. 337p. 